\title{
PERIODIC SOLUTIONS \\ FOR NONLINEAR EVOLUTION EQUATIONS IN A BANACH SPACE
}

\author{
IOAN I. VRABIE
}

(Communicated by Kenneth R. Meyer)

\begin{abstract}
We prove an existence result for $T$-periodic mild solutions to nonlinear evolution equations of the form

$$
u^{\prime}(t)+A u(t) \ni F(t, u(t)), \quad t \in R_{+} .
$$

Here $(X,\|\cdot\|)$ is a real Banach space, $A: D(A) \subset X \rightarrow 2^{X}$ is an operator with $A-a I m$-accretive for some $a>0$ and such that $-A$. generates a compact semigroup, while $F: R_{+} \times \overline{D(A)} \rightarrow X$ is a Carathéodory mapping which is $T$-periodic with respect to its first argument and satisfies

$$
\lim _{r \rightarrow+\infty} \frac{1}{r} \sup \left\{\|F(t, v)\| ; t \in R_{+}, v \in \overline{D(A)},\|v\| \leq r\right\}<a .
$$

As a consequence, we obtain an existence theorem for $T$-periodic solutions to the porous medium equation.
\end{abstract}

\section{INTRODUCTION}

The main goal of the present paper is to study the existence of $T$-periodic mild solutions to a class of nonlinear evolution equations of the form

$$
u^{\prime}(t)+A u(t) \ni F(t, u(t)), \quad t \in R_{+} .
$$

Here $(X,\|\cdot\|)$ is a real Banach space, $A: D(A) \subset X \rightarrow 2^{X}$ is a nonlinear operator, and $F: R_{+} \times \overline{D(A)} \rightarrow X$ is a Carathéodory mapping, i.e., for each $u \in \overline{D(A)}$ the function $t \mapsto F(t, u)$ is measurable, and for each $t \in R_{+}$the function $u \mapsto F(t, u)$ is continuous. Namely, we will prove

Theorem 1. Assume that $\overline{D(A)}$ is convex, there exists a>0 such that $A-a I$ is $m$-accretive, and $-A$ generates a compact semigroup. Assume further that $F$ is a Carathéodory mapping which is $T$-periodic with respect to its first argument and satisfies

$$
\lim _{r \rightarrow+\infty} \frac{1}{r} \sup \left\{\|F(t, v)\| ; t \in R_{+}, v \in \overline{D(A)},\|v\| \leq r\right\}=m<a .
$$

Then equation (1.1) has at least one $T$-periodic mild solution.

Received by the editors June 19, 1988 and, in revised form, January 27, 1989.

1980 Mathematics Subject Classification (1985 Revision). Primary 34G20, 34C25; Secondary $35 \mathrm{~K} 55$.

Key words and phrases. Accretive operator, compact semigroups, periodic solution, porous medium equation. 
The most common approach for solving (1.1) in the class of $T$-periodic functions is to look for a fixed point of the corresponding Poincare map. We recall that this is the possible multivalued map $P$ which assigns to each $x \in$ $\overline{D(A)}$ the values at $T$ of all mild solutions $u$ of (1.1) satisfying $u(0)=x$. But in order to appeal to a fixed point theorem applying to multivalued mappings, one has first to verify either that $P$ is convex-valued or that it is a contraction with respect to the Hausdorff-Pompeiu metric. Since both these conditions are very hard to check whenever $P$ is multivalued, one usually assumes that $P$ is single-valued. This method has been followed by many authors and, so far as the infinite-dimensional case is concerned, one of the first steps in this direction is due to Browder [6]. In [6], $A=A(t)$ is a family of closed linear accretive operators acting in a Hilbert space $H$, and $-F(t, \cdot)$ is a family of nonlinear accretive operators in $H$. Under these assumptions, one shows that $P$ satisfies the conditions in the Browder-Göhde-Kirk fixed point theorem [ 5 , Théorème 1.2 , p. 5]. For related results see [5, Théorème 3.15 , p. 95; 2, Theorem 2.4 , p. 138 and Corollary 3.4 , p. 160].

The next step was to discard the accretiveness assumption on $-F(t, \cdot)$. With this aim, using an interplay among accretiveness, compactness, and flowinvariance techniques, Lightbourne [9, Theorem 3] solves (1.1) for the class of $T$-periodic functions in the case in which $A=A(t)$ is a family of continuous accretive operators from a closed bounded and convex subset $D$ in $X$ into $X, F$ is a compact mapping from $R_{+} \times D$ into $X$, and $-A+F$ satisfies a Nagumo-type tangential condition with respect to $D$. However, the continuity of $A(t)$ from $D$ into $X$ precludes the applicability of Lightbourne's result directly to partial differential equations. The same remark holds true also for the results in Deimling [8] which are closely related to those of Lightbourne [9]. A fixed point device for the Poincaré map applied to partial differential equations may be found in Lions [10, p. 483].

For a generic property of (1.1) with respect to $T$-periodic solutions, see Benavides [4] and the references therein.

If $F$ is merely continuous with respect to its second argument and $-A$ generates a compact semigroup one knows that, for each $x \in \overline{D(A)}$, equation (1.1) has at least one (local) mild solution $u$ satisfying $u(0)=x$, but a nonuniqueness phenomenon may occur. (See [13] or [15, Theorem 3.8.2, p. 180].) Therefore, in this case, inasmuch as $P$ is not single-valued, we have to adopt a different strategy. The first attempt to solve (1.1) in these circumstances was made by Prüss [12], who has studied a semilinear version of (1.1). Namely, in [12, Theorem 3(i)], $A$ is a linear densely defined operator acting in $X, D$ is a closed convex and bounded subset in $X, F: R_{+} \times D \rightarrow X$ is a continuous, bounded function and, again as in $[8,9],-A+F$ satisfies a Nagumo-type tangential condition with respect to $D$. Assuming that $D$ has nonempty interior and $-A$ generates a compact semigroup, Prüss [12, Theorem 3(i)] proves that a suitably defined family of operators satisfies the hypotheses of the Leray- 
Schauder fixed point theorem, and thus (1.1) has at least one $T$-periodic mild solution.

Subsequently, Becker [3] considered the case in which $A$ is a closed densely defined linear operator acting in a real separable Hilbert space $H$ such that - $A$ generates a compact semigroup, while $F: R_{+} \times H \rightarrow H$ is of the form $F(t, u)=-B(t, u) u+f(t, u)$ for $(t, u)$ in $R_{+} \times H$, where $B: R_{+} \times H \rightarrow L(H)$ and $f: R_{+} \times H \rightarrow H$ are Carathéodory mappings which are uniformly bounded on $R_{+} \times H$. Using the extra condition that (in our terminology) $A-a I$ is $m$ accretive for some $a>0$ and $\sup \left\{\|B(t)\|_{L(H)} ; t \in R_{+}\right\}<a$, he shows that, for each $v \in L^{2}([0, T] ; H)$, the equation

$$
u^{\prime}(t)+A u(t)=F(t, v(t)), \quad t \in[0, T]
$$

has a unique mild solution $u=M(v)$ satisfying $u(0)=u(T)$. Finally, Becker [3, Theorem 3.1] proves that the operator $v \mapsto M(v)$ defined as before satisfies the hypotheses of Schauder's fixed point theorem.

As we can easily see, Theorem 1.1 extends Becker's result to general Banach spaces and to fully nonlinear operators $A$ and $F$ and, so far as the condition on the operator $A$ is concerned, that of Prüss [12, Theorem 3(i)]. We note that, by using similar arguments as in Vrabie [14], we can prove a more general result which completely incorporates the case considered in Prüss [12, Theorem 3(i)]-where $F$ is defined merely on $R_{+} \times D$ and not on $R_{+} \times \overline{D(A)}$-but we refrain from doing so here in order to avoid distracting technicalities.

Since the compactness of the semigroup generated by $-A$ is a parabolic condition (see [15, Example 2.2.1, p. 51 and Example 2.2.2, p. 52]) our main result is applicable to various classes of nonlinear partial differential equations of parabolic type including the porous medium equation.

For an appropriate variant applying to nonlinear hyperbolic problems, the interested reader is referred to Vrabie [16].

Although we also use a fixed point device, our method of proof is different from that in [3] or [12], which is specific to the semilinear case, where each mild solution of (1.1) can be expressed by means of the variation of constants formula.

\section{Preliminaries}

We assume familiarity with the basic concepts and results concerning $m$ accretive operators and mild solutions, and we refer the reader to Barbu [2, Chapter III], Crandall [7], Pavel [11, Chapter 1], or Vrabie [15, Chapter 1] for an introduction to this subject.

Let $(X,\|\cdot\|)$ be a real Banach space and let $A: D(A) \subset X \rightarrow 2^{X}$ be an operator with $A-a I m$-accretive for some $a>0$. It is well known that, in these circumstances, for each $(x, f) \in \overline{D(A)} \times L^{1}([0, T] ; X)$, the Cauchy 
problem

$$
\begin{aligned}
& u^{\prime}(t)+A u(t) \ni f(t), \quad t \in[0, t], \\
& u(0)=x
\end{aligned}
$$

has a unique mild (integral) solution $u=M(x, f)$. In addition, for each $\left(x_{i}, f_{i}\right) \in \overline{D(A)} \times L^{1}([0, T] ; X), u_{i}=M\left(x_{i}, f_{i}\right) \quad(i=1,2)$ satisfies

$$
\left\|u_{1}(t)-u_{2}(t)\right\| \leq e^{-a(t-s)}\left\|u_{1}(s)-u_{2}(s)\right\|+\int_{s}^{t} e^{-a(t-\tau)}\left\|f_{1}(\tau)-f_{2}(\tau)\right\| d \tau
$$

for $0 \leq s \leq t \leq T$. (See [2, Theorem 2.1, p. 124, and Remark, p. 131; 7; 11, Chapter 1; or 15, Chapter 1].)

The semigroup $\{S(t) ; t \geq 0\}$ generated by $-A$ on $\overline{D(A)}$ via the Crandall and Liggett exponential formula is called compact if, for each $t>0, S(t)$ is a compact operator.

A subset $G$ in $L^{1}([0, T] ; X)$ is called uniformly integrable if

$$
\lim _{\mu(E) \downarrow 0} \int_{E}\|f(t)\| d t=0
$$

uniformly for $f \in G$, where, for each measurable subset $E$ in $[0, T], \mu(E)$ denotes the Lebesgue measure of $E$.

The main ingredient in the proof of Theorem 1.1 is the following slight extension of some previous results due to Baras [1] and Vrabie [13].

Theorem 2. If $A: D(A) \subset X \rightarrow 2^{X}$ is an operator with $A$-aI $m$-accretive for some $a \geq 0$ such that $-A$ generates a compact semigroup, then for each bounded subset $B$ in $\overline{D(A)}$ and each uniformly integrable subset $G$ in $L^{1}([0, T] ; X)$, the set $M(B \times G)$ of all mild solutions of (2.1) corresponding to $(x, f) \in B \times G$ is relatively compact in $C([d, T] ; X)$ for each $d \in(0, T)$. If, in addition, $B$ is relatively compact in $X$, then $M(B \times G)$ is relatively compact in $C([0, T] ; X)$.

Since the proof of Theorem 2 follows-except for some obvious modifications-the same lines as in the proof of [15, Theorem 2.3.2, p. 64], we do not give details.

\section{Proof of TheOREM 1}

We begin with two simple remarks which will prove useful later.

Remark 3.1. If $F: R_{+} \times \overline{D(A)} \rightarrow X$ is a Carathéodory mapping satisfying (1.2), then it maps bounded subsets in $R_{+} \times \overline{D(A)}$ into bounded subsets in $X$. Therefore, for each continuous function $u:[0, T] \rightarrow \overline{D(A)}$, the superposition mapping $t \mapsto F(t, u(t))$ belongs to $L^{\infty}([0, T] ; X)$.

Remark 3.2. In the hypotheses of Theorem 1, we may assume with no loss of generality that $0 \in D(A)$ and $0 \in A 0$. Indeed, if this is not the case, fix $x \in D(A), y \in A x$ and define $u:=v+x, \tilde{A} v:=A(v+x)-y$, and 
$\widetilde{F}(t, v):=F(t, v+x)-y$. Then $(1.1)$ may be equivalently written as

$$
v^{\prime}(t)+\widetilde{A} v(t) \ni \widetilde{F}(t, v(t)), \quad t \in R_{+},
$$

where $\widetilde{A}$ and $\widetilde{F}$ satisfy all the hypotheses of Theorem 1.1 and, in addition, $0 \in D(\widetilde{A})$ and $0 \in \widetilde{A} 0$.

The idea of the proof consists in showing that a suitably defined operator has at least one fixed point which is a $T$-periodic mild solution to (1.1). We begin with the definition of this operator. First, let $C([0, T] ; \overline{D(A)})$ be the set of all continuous functions $u:[0, T] \rightarrow \overline{D(A)}$, and let us define the operator $J: C([0, T] ; \overline{D(A)}) \rightarrow \overline{D(A)}$ by

$$
J u:=z(T)
$$

for each $u \in C([0, T] ; \overline{D(A)})$, where $z$ is the unique mild solution of the Cauchy problem

$$
\begin{aligned}
& z^{\prime}(t)+A z(t) \ni F(t, u(t)), \quad t \in[0, T] \\
& z(0)=u(0) .
\end{aligned}
$$

Next, let us define the operator $Q: C([0, T] ; \overline{D(A)}) \rightarrow C([0, T] ; \overline{D(A)})$ by

$$
Q u:=v
$$

for each $u \in C([0, T] ; \overline{D(A)})$, where $v$ is the unique mild solution of the Cauchy problem

$$
\begin{aligned}
& v^{\prime}(t)+A v(t) \ni F(t, u(t)), \quad t \in[0, T] \\
& v(0)=J u .
\end{aligned}
$$

Since, by Remark 3.1, for each $u \in C([0, T] ; \overline{D(A)}), t \mapsto F(t, u(t))$ belongs to $L^{\infty}([0, T] ; X)$ which is included in $L^{1}([0, T] ; X)$ and $u(0) \in \overline{D(A)}$, both $J$ and $Q$ are well defined.

At this point, let us observe that (1.1) has at least one $T$-periodic mild solution if and only if $Q$ has at least one fixed point. Indeed, if $u$ is a $T$-periodic mild solution to $(1.1)$, then $J u=u(T)=u(0)$, and consequently the unique mild solution $v$ of (3.2) must coincide with $u$, i.e., $Q u=u$. Conversely, if $u$ is a fixed point of $Q$, then $u$ and $z$ are mild solutions of (3.1) corresponding to the same initial data: $u(0)$ and $F(\cdot, u(\cdot))$, and thus, by $(2.2), u=z$. Hence, taking into account (3.1) and (3.2), it readily follows that $u$ is a $T$-periodic mild solution of (1.1).

Thus, it is clear that, in order to complete the proof, it suffices to show that $Q$ has at least one fixed point. We do this with the help of the following two lemmas.

Lemma 3.1. Assume that there exists a>0 such that $A$-aI is $m$-accretive, $0 \in D(A)$ and $0 \in A 0$. Assume further that $F$ is a Caratheodory mapping which satisfies (1.2). Then there exists $r>0$ such that the operator $Q$, defined as before, maps the set

$$
B(0, r):=\{u \in C([0, T] ; \overline{D(A)}) ;\|u(t)\| \leq r, t \in[0, T]\}
$$

into itself. 
Lemma 3.2. Assume that all the hypotheses of Theorem 1.1 are satisfied, $0 \in$ $D(A)$ and $0 \in A 0$. Then, for each $r>0, Q(B(0, r))$ is relatively compact in $C([0, T] ; X)$, where $B(0, r)$ is given by (3.3).

Proof of Lemma 3.1. Let us assume by contradiction that for each $r>0$ $Q$ does not map $B(0, r)$ into itself. Then there exists a sequence $\left(u_{n}\right)$ in $C([0, T] ; \overline{D(A)})$ such that

$$
\left\|u_{n}\right\|_{C} \leq n \quad \text { and } \quad\left\|Q u_{n}\right\|_{C}>n
$$

for each $n \in N$, where $\|\cdot\|_{C}$ is the usual norm of $C([0, T] ; X)$. Since $0 \in A 0$ and $Q u_{n}$ verifies (3.2) for $u=u_{n}$, from (2.2) we get

$$
\left\|\left(Q u_{n}\right)(t)\right\| \leq e^{-a t}\left\|J u_{n}\right\|+\int_{0}^{t} e^{-a(t-s)}\left\|F\left(s, u_{n}(s)\right)\right\| d s
$$

for each $n \in N$ and $t \in[0, T]$. For each $n \in N$ let us choose $t_{n} \in[0, T]$ satisfying

We note that

$$
\left\|Q u_{n}\right\|_{C}=\left\|\left(Q u_{n}\right)\left(t_{n}\right)\right\|
$$

$$
\left(t_{n}\right) \text { has some subsequence tending to a limit } T_{0} \in[0, T] \text {. }
$$

Next, recalling the definition of $J$ and the fact that $0 \in A 0,(2.2)$ yields

$$
\left\|J u_{n}\right\| \leq e^{-a T}\left\|u_{n}(0)\right\|+\int_{0}^{T} e^{-a(T-s)}\left\|F\left(s, u_{n}(s)\right)\right\| d s
$$

for each $n \in N$. This inequality, along with (3.5), leads to

$$
\begin{gathered}
\left\|Q u_{n}\right\|_{C} \leq e^{-a t_{n}}\left[e^{-a T}\left\|u_{n}\right\|_{C}+\frac{1-e^{-a T}}{a}\left\|F\left(\cdot, u_{n}(\cdot)\right)\right\|_{\infty}\right] \\
+\frac{1-e^{-a t_{n}}}{a}\left\|F\left(\cdot, u_{n}(\cdot)\right)\right\|_{\infty}
\end{gathered}
$$

for each $n \in N$, where $\|\cdot\|_{\infty}$ is the usual norm of $L^{\infty}([0, T] ; X)$.

From (3.4) and (3.7), we then have

$$
\begin{aligned}
1<\frac{\left\|Q u_{n}\right\|_{C}}{n} \leq & \frac{\left\|Q u_{n}\right\|_{C}}{\left\|u_{n}\right\|_{C}} \\
\leq & \frac{e^{-a t_{n}}}{\left\|u_{n}\right\|_{C}}\left[e^{-a T}\left\|u_{n}\right\|_{C}+\frac{1-e^{-a T}}{a}\left\|F\left(\cdot, u_{n}(\cdot)\right)\right\|_{\infty}\right] \\
& \quad+\frac{1-e^{-a t_{n}}}{a\left\|u_{n}\right\|_{C}}\left\|F\left(\cdot, u_{n}(\cdot)\right)\right\|_{\infty}
\end{aligned}
$$

for each $n \in N$. From (3.4), (3.7), and Remark 3.1, it readily follows that $\lim _{n}\left\|u_{n}\right\|_{C}=+\infty$. Then, passing to the limsup for $n \rightarrow+\infty$ in (3.8) and using (3.6) and (1.2), we get

$$
1 \leq e^{-a T_{0}} e^{-a T}+\frac{m}{a} \cdot e^{-a T_{0}}\left(1-e^{-a T}\right)+\frac{m}{a}\left(1-e^{-a T_{0}}\right)<1
$$


This contradiction shows that the initial supposition is false, and thus the proof of Lemma 3.1 is complete.

Proof of Lemma 3.2. First, let us observe that, in view of Remark 3.1, the set $G=\{F(\cdot, u(\cdot)) ; u \in B(0, r)\}$ is uniformly integrable in $L^{1}([0, T] ; X)$. From Theorem 2.1, it then follows that the set $B=\{J u: u \in B(0, r)\}$ is relatively compact in $X$. Using once again Theorem 2.1, we conclude that $M(B \times G)$-the set of all mild solutions of $(1.1)$ corresponding to $(x, f) \in B \times G$-is relatively compact in $C([0, T] ; X)$. But $Q(B(0, r))$ is obviously included in $M(B \times G)$, and therefore it is relatively compact in $C([0, T] ; X)$, as claimed.

Proof of Theorem 1.1. In view of Remark 3.2 we may always assume that $0 \in D(A)$ and $0 \in A 0$, and consequently Lemma 3.1 applies. Clearly, the set $B(0, r)$ given by (3.3) is nonempty, bounded, closed, and convex. Since $Q$ maps $B(0, r)$ into itself and, by Lemma 3.2, $Q(B(0, r))$ is relatively compact in $C([0, T] ; X)$, in order to appeal to Schauder's fixed point theorem it suffices to check the continuity of $Q$. To this end, let us observe that, from the definition of $J$, that of $Q$, and (2.2), we have

$$
\begin{aligned}
\|(Q u)(t)-(Q \tilde{u})(t)\| \leq & e^{-a T}\|u(0)-\tilde{u}(0)\| \\
& +\int_{0}^{T} e^{-a(T-s)}\|F(s, u(s))-F(s, \tilde{u}(s))\| d s \\
& +\int_{0}^{t} e^{-a(t-s)}\|F(s, u(s))-F(s, \tilde{u}(s))\| d s
\end{aligned}
$$

for each $u, \tilde{u} \in B(0, r)$ and $t \in[0, T]$. Then, by Remark 3.1 and the Lebesgue dominated convergence theorem, we easily conclude that $Q$ is continuous from $B(0, r)$ into itself. Finally, by Schauder's fixed point theorem, $Q$ has at least one fixed point, and this completes the proof of Theorem 1.1.

\section{AN EXAMPLE}

Let $\Omega$ be a bounded domain in $R^{n}, n \geq 2$, with sufficiently smooth boundary $\Gamma$, and let us consider the following periodic problem for the porous medium equation

$$
\begin{aligned}
& \frac{\partial u}{\partial t}-\Delta \rho(u)+a u=f(t, x, u) \quad \text { a.e. for }(t, x) \in R_{+} \times \Omega, \\
& u=0 \text { a.e. for }(t, x) \in R_{+} \times \Gamma, \\
& u(t, x)=u(t+T, x) \quad \text { for each } t \in R_{+} \text {and a.e. for } x \in \Omega .
\end{aligned}
$$

Theorem 4. If $\rho \in C(R ; R) \cap C^{1}(R-\{0\} ; R), \rho(0)=0$ and there exist $c>0$ and $p>\max \left\{\frac{n-2}{2}, 0\right\}$ such that $\rho^{\prime}(r) \geq c|r|^{p-1}$ for each $r \in R-\{0\}, a>0$, and $f: R_{+} \times \Omega \times R \rightarrow R$ is continuous, and $T$-periodic with respect to its first argument and there exist $m \in(0, a)$ and $b \geq 0$ such that

$$
|f(t, x, u)| \leq m|u|+b \text { for each }(t, x, u) \in R_{+} \times \bar{\Omega} \times R,
$$


then (4.1) has at least one solution $u$ satisfying

(4.3) $u \in C\left(R_{+} ; L^{1}(\Omega)\right)$,

(4.4) $t \mapsto \frac{\partial u}{\partial t}$ belongs to $L^{2}\left([0, T] ; H^{-1}(\Omega)\right)$ and $t \mapsto \rho(u)$ belongs to $L^{2}\left([0, T] ; H_{0}^{1}(\Omega)\right)$,

(4.5) $t \mapsto \int_{\Omega} j(u) d x$ belongs to $A C\left(R_{+} ; R\right)$, where $j(r):=\int_{0}^{r} \rho(s) d s$ for each $r \in R$.

Proof. Take $X=L^{1}(\Omega)$ and let us define $A: D(A) \subset X \rightarrow 2^{X}$ by

$$
A u:=\{-\Delta \rho(u)+a u\}
$$

for each $u \in D(A):=\left\{u \in L^{1}(\Omega) ; \rho(u) \in W_{0}^{1,1}(\Omega), \Delta \rho(u) \in L^{1}(\Omega)\right\}$. Next, let us define $F: R_{+} \times L^{1}(\Omega) \rightarrow L^{1}(\Omega)$ by

$$
(F(t, u))(x):=f(t, x, u(x))
$$

for each $u \in L^{1}(\Omega), t \in R_{+}$and a.e. for $x \in \Omega$. At this point, let us observe that (4.1) may be rewritten as an abstract evolution $T$-periodic problem of the form $(1.1)$ in $L^{1}(\Omega)$. It is known that $-A$ generates a compact semigroup on $\overline{D(A)}=L^{1}(\Omega)$ (see [15, Lemma 2.6.2, p. 95]) and $A-a I$ is $m$-accretive (see [15, Example 1.5 .5 , p. 24]). From (4.2) it readily follows that $F$ is continuous from $R_{+} \times L^{1}(\Omega)$ into $L^{1}(\Omega)$, is $T$-periodic with respect to its first argument, and satisfies (1.2). Then, by Theorem 1.1, the equation (1.1) with $A$ and $F$ as above has at least one $T$-periodic mild solution satisfying (4.3). Since (4.4) and (4.5) follow exactly as in the proof of [15, Theorem 3.9.1, p. 184], the proof is complete.

\section{ACKNOWLEDGMENT}

The author would like to express his warmest thanks to the referee for useful suggestions and comments concerning the presentation.

\section{REFERENCES}

1. P. Baras, Compacité de l'opérateur $f \mapsto u$ solution d'une équation non-linéaire $(d u / d t)+$ $A u \ni f$, C. R. Acad. Sci. Paris 286 (1978), 1113-1116.

2. V. Barbu, Nonlinear semigroups and differential equations in Banach spaces, Editura Academiei Bucureşti, Noordhoff, 1976.

3. R. I. Becker, Periodic solutions of semilinear equations of evolution of compact type, J. Math. Anal. Appl. 82 (1981), 33-48.

4. T. D. Benavides, Generic existence of periodic solutions of differential equations in Banach spaces, Bull. Pol. Acad. Sci. (Math.) 33 (1985), 129-135.

5. H. Brézis, Opérateurs maximaux monotones et semi-groupes de contractions dans un espace de Hilbert, North-Holland, 1973. 
6. F. E. Browder, Existence of periodic solutions for nonlinear equations of evolution, Proc. Nat. Acad. Sci. U.S.A. 53 (1965), 1100-1103.

7. M. G. Crandall, Nonlinear semigroups and evolution governed by accretive operators, Proc. Sympos. Pure Math., vol. 45, Part 1, Amer. Math. Soc., Providence, RI, 1986, pp. 305-337.

8. K. Deimling, Periodic solutions of differential equations in Banach spaces, Manuscripta Math. 24 (1978), 31-44.

9. J. H. Lightbourne III, Periodic solutions for a differential equation in Banach space, Trans. Amer. Math. Soc. 238 (1978), 285-299.

10. J. L. Lions, Quelques méthodes de résolution des problèmes aux limites non-linéaires, Gauthier-Villars, Paris, 1969.

11. N. H. Pavel, Nonlinear evolution operators and semigroups. Applications to partial differential equations, Lecture Notes in Math., vol. 1260, Springer-Verlag, Berlin and New York, 1987.

12. J. Prüss, Periodic solutions of semilinear evolution equations, Nonlinear Anal. T.M.A. 3 (1979), 601-612.

13. I. I. Vrabie, The nonlinear version of Pazy's local existence theorem, Israel J. Math. 32 (1979), 221-235.

14. __ Compactness methods and flow-invariance for perturbed nonlinear semigroups, An. Stiint. Univ. “Al. I. Cuza” Iaşi Secţ. I a Mat. 27 (1981), 119-125.

15. __ Compactness methods for nonlinear evolutions, Pitman Monographs and Surveys in Pure and Applied Mathematics, vol. 32, Longman Scientific \& Technical, 1987.

16. __ Periodic solutions of nonlinear evolution equations in a Hilbert space, in preparation.

Department of Mathematics, Polytechnic Institute of Iasi, 6600, Romania

Current address: P. O. Box 180, Ro, Iş 1, Iaşi 6600, Romania 